\title{
Average Contiguous Duration - A Novel Metric for Characterizing Wireless Fading Channels
}

\author{
Syed Junaid Nawaz, Muhammad Adil, and Shurjeel Wyne
}

\begin{abstract}
We define the average contiguous duration (ACD) of the received fading signal as the average time duration during which the signal envelope contiguously remains within a bounded amplitude interval. Also, the multi-interval ACD or $K$-ACD function is defined as the set of ACD values for $K$ non-overlapping amplitude-intervals whose union spans the received envelope's amplitude range. We derive closed-form expressions of the ACD for Rayleigh, Rice, and Nakagami$m$ fading signals, which are widely analyzed in the literature. The derived expressions hold practical significance for several signal processing applications such as non-uniform quantization of channel samples for secret key generation (SKG) in physical layer security (PLS) techniques. The well-known channel fading metric of average fade duration (AFD) is shown as a special case of the proposed ACD metric and the proposed theoretical analysis is validated by simulations.
\end{abstract}

Index Terms-Average fade duration, characterization, contiguous duration, fading channel

\section{INTRODUCTION}

Various metrics have been discussed in the literature to quantify the behaviour of fading signals. The probability distribution function (PDF) of the received signal's envelope can be used to predict the likelihood of the envelope residing in a certain interval. The standard deviation (SD), variance, and root mean square (RMS) metrics quantify the signal dispersion in relation to its mean. To quantify the envelope fluctuation rate, the level-crossing rate (LCR) and averagefade duration (AFD) metrics are widely used [1]. The LCR is the average rate at which the signal envelope crosses a given threshold-level with a positive or a negative slope. The AFD is the average duration for which the signal envelope remains below some given threshold-level. The AFD is suitable for applications where signal fluctuations relative to a single threshold are of interest, e.g., to compare signal strength with the minimum threshold for reliable communication. However, in other signal processing applications such as quantization and encoding, signal fluctuations may need to be quantified in relation to multiple thresholds simultaneously. In this context, this letter proposes a novel metric termed average contiguous duration $(A C D)$, which is the average time duration for which a given signal's envelope contiguously remains within a bounded amplitude interval. Moreover, the multi-interval ACD or $K$ ACD function is defined as the set of ACD values for $K$ non-overlapping amplitude-intervals whose union spans the

S. J. Nawaz, M. Adil, and S. Wyne are with the Department of Electrical and Computer Engineering, COMSATS University Islamabad (CUI), 45550 Islamabad, Pakistan. (email: junaidnawaz@ieee.org, adil34700@gmail.com, and shurjeel.wyne@comsats.edu.pk)

This work has been submitted to the IEEE for possible publication. Copyright may be transferred without notice, after which this version may no longer be accessible. received envelope's amplitude range. The ACD metric has uses in several signal processing applications such as in determining appropriate non-uniform quantization (NUQ) intervals for the channel samples in secret key generation (SKG) for physical layer security (PLS). The ACD-based NUQ intervals can offer promising trade-offs between the key generation rate (KGR), key agreement probability (KAP), and key randomness property (KRP). The rest of this letter is organized as follows: In Sec. II, the proposed ACD metric is defined and closed-form expressions of ACD for the Rayleigh, Rice, and Nakagami- $m$ distributions are derived. In Sec. III, the multilevel $K$-ACD metric is defined. In Sec. IV, applications of the proposed metrics are highlighted with emphasis on SKG for PLS. Finally, Sec. V concludes this letter.

\section{Average Contiguous Duration (ACD)}

We define ACD as the average time duration for which the received signal envelope $|r(t)|$ remains within an amplitude range of interest (ARoI) that is bounded by two threshold levels $\rho_{1}$ and $\rho_{2}$. Fig. 1 shows $M$ contiguous duration (CD) instances, $\xi_{m} m=1,2 \ldots, M$. For this case the ACD is obtained by averaging as $\Xi_{\rho_{1}}^{\rho_{2}}=\frac{1}{M} \sum_{m=1}^{M} \xi_{m}$. The ACD can be defined more rigorously as

$$
\Xi_{\rho_{1}}^{\rho_{2}}=\frac{1}{\mathcal{M}} \operatorname{Prob}\left(\rho_{1}<|r| \leq \rho_{2}\right),
$$

where $\mathcal{M}$ represents the rate (CD instances per second) at which the signal envelope enters the ARoI with either a positive or a negative slop. In Fig. 1 the start and end points of each of the $M=7 \mathrm{CD}$ instances is marked with $\circ$ and $\bullet$, respectively. A CD may start with the signal envelope entering the ARoI with a negative slope from an amplitude greater than $\rho_{2}$ or with a positive slope from an amplitude smaller than $\rho_{1}$. The level-crossing rate (LCR) of the signal envelope with reference to some threshold $\rho_{(.)}$is the average number of times per second that the envelope crosses $\rho_{(.)}$with a positive or a negative slope $\left(N_{r}^{+}\left(\rho_{(.)}\right)=N_{r}^{-}\left(\rho_{(.)}\right)\right)$[2]. The LCR can be expressed as $N_{r}(\rho)=\int_{0}^{\infty} \dot{r} f(\rho, \dot{r}) \mathrm{d} \dot{r}$, where $\dot{r}$ represents the time derivative of the signal envelope $r, \rho$ is the predefined threshold, and $f(.,$.$) represents the joint density function. The$ parameter $\mathcal{M}$ can be interpreted as a sum of the LCR for $\rho_{2}$ (with negative slope) and the LCR for $\rho_{1}$ (with positive slope), i.e., $\mathcal{M}=N_{r}^{-}\left(\rho_{1}\right)+N_{r}^{+}\left(\rho_{2}\right)$. Then the ACD from (1) can be expressed as

$$
\Xi_{\rho_{1}}^{\rho_{2}}=\frac{1}{N_{r}\left(\rho_{1}\right)+N_{r}\left(\rho_{2}\right)} \int_{\rho_{1}}^{\rho_{2}} f(r) \mathrm{d} r=\frac{F_{r}\left(\rho_{2}\right)-F_{r}\left(\rho_{1}\right)}{N_{r}\left(\rho_{2}\right)+N_{r}\left(\rho_{1}\right)} .
$$

where $f(r), F_{r}\left(\rho_{(.)}\right), N_{r}\left(\rho_{(.)}\right)$represent the PDF, CDF, and LCR of signal envelope, respectively. The Average fade 


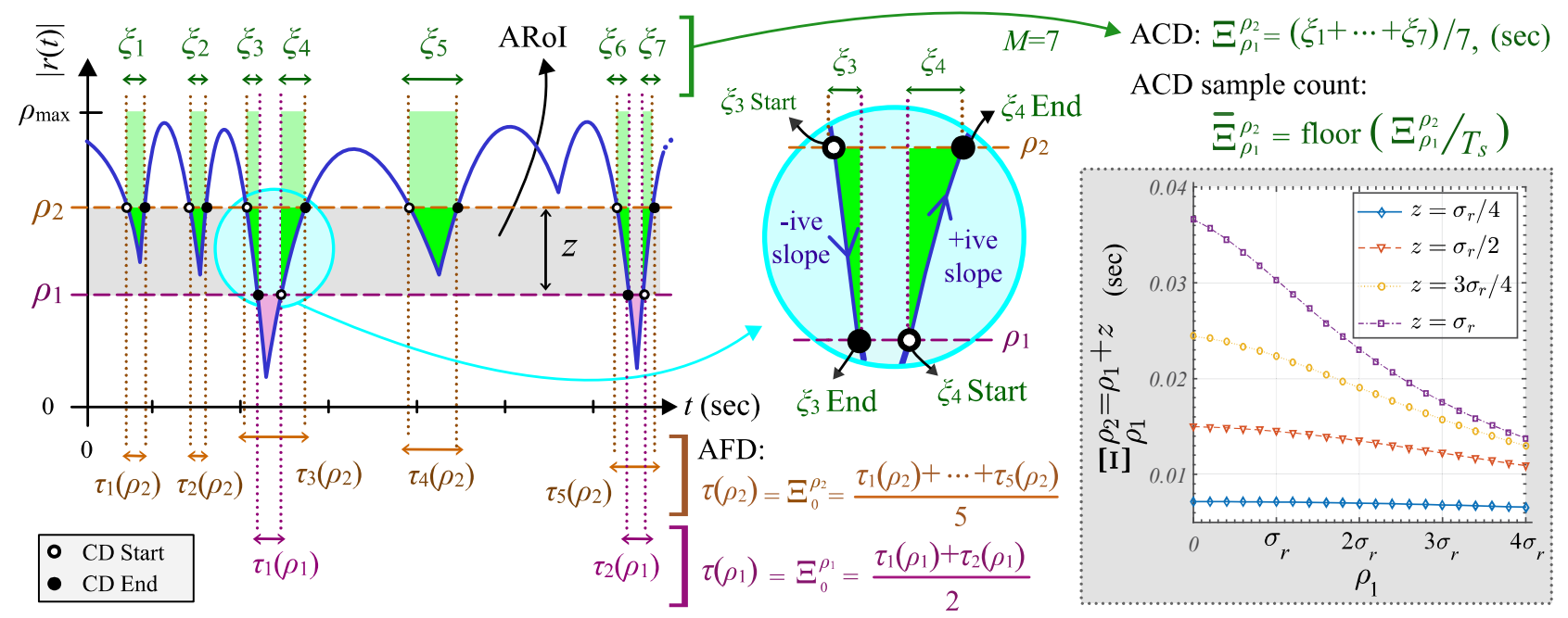

Fig. 1: Demonstration of ACD and AFD calculations for a sample function of Rayleigh fading signal.

duration (AFD), $\tau_{r}(\rho)$, represents the average duration for which the signal envelope stays below a given threshold level $\rho$, i.e, it is the ratio between the total time the envelope remains below the threshold and the total number of times the envelope crosses the threshold with positive or a negative slope. The AFD can be obtained as a special case of the ACD by substituting $\rho_{2}=\rho$ and $\rho_{1}=0$. The AFD can be expressed in terms of the ACD as

$$
\tau_{r}(\rho)=\Xi_{0}^{\rho}=\frac{\operatorname{Prob}(0<r \leq \rho)}{N_{r}(\rho)+N_{r}(0)}=\frac{1}{N_{r}(\rho)} \int_{0}^{\rho} f(r) \mathrm{d} r=\frac{F_{r}(\rho)}{N_{r}(\rho)} .
$$

The ACD metric can help determine the appropriate sampling rate to ensure that a certain number of contiguous signal samples fall within the ARoI. For example, by setting the sampling interval as $T_{s}=\Xi_{\rho_{1}}^{\rho_{2}} / N$, on average $N$ contiguous samples will lie within the ARoI $\rho_{2}-\rho_{1}$. Alternatively, an ACD value can be converted to an integer sample-count as $\bar{\Xi}_{\rho_{1}}^{\rho_{2}}=\operatorname{floor}\left(\Xi_{\rho_{1}}^{\rho_{2}} / T_{s}\right)$.

For reference, Table I [2] lists the mathematical expressions of the PDF, CDF, LCR, and AFD for Rayleigh, Rice, and Nakagami- $m$ distributed signal envelopes and we provide their closed-form ACD expressions below.

a) Rayleigh: By substituting the expressions of Rayleigh CDF and LCR from Table I into (2) and after simplification the ACD for Rayleigh signal envelope can be expressed as

$$
\Xi_{\rho_{1}}^{\rho_{2}}=\frac{\sqrt{\Omega}\left(\exp \left(\frac{\rho_{2}^{2}}{\Omega}\right)-\exp \left(\frac{\rho_{1}^{2}}{\Omega}\right)\right)}{f_{m} \sqrt{2 \pi}\left(\rho_{1} \exp \left(\frac{\rho_{2}^{2}}{\Omega}\right)+\rho_{2} \exp \left(\frac{\rho_{1}^{2}}{\Omega}\right)\right)},
$$

where $f_{m}$ is the maximum Doppler shift, $\Omega=E\left[r^{2}\right]=$ $2 \sigma_{r}^{2}$ represents the average signal power, $\sigma_{r}$ is the standard deviation of signal amplitude, and $E[$.$] denotes statistical$ expectation.

b) Rice: By substituting Rician CDF and LCR expressions from Table I into (2) and after simplifying the ACD for Rician case can be expressed as

$$
\begin{aligned}
& \Xi_{\rho_{1}}^{\rho_{2}}=\frac{\Omega \exp (k)}{f_{m} \sqrt{2 \pi(k+1)}}\left\{Q_{1}\left(\sqrt{2 k}, \sqrt{\frac{2 \rho_{1}^{2}(k+1)}{\Omega}}\right)\right. \\
& \left.-Q_{1}\left(\sqrt{2 k}, \sqrt{\frac{2 \rho_{2}^{2}(k+1)}{\Omega}}\right)\right\}\left\{I_{0}\left(2 \rho_{1} \sqrt{\frac{k(k+1)}{\Omega}}\right)\right. \\
& \left.\quad \times \rho_{1} \exp \left(-\frac{\rho_{1}^{2}(k+1)}{\Omega}\right)+I_{0}\left(2 \rho_{2} \sqrt{\frac{k(k+1)}{\Omega}}\right) \rho_{2} \exp \left(-\frac{\rho_{2}^{2}(k+1)}{\Omega}\right)\right\}^{-1}
\end{aligned}
$$

where $k \geq 0$ is the Rice parameter, $Q_{(.)}(.,$.$) is the Marcum- Q$ function, and $I_{\circ}($.$) is the 0^{\text {th }}$-order modified Bessel function of first-kind.

c) Nakagami-m: By substituting the Nakagami- $m$ CDF and LCR expressions from Table I into (2) the Nakagami-m ACD can be expressed as

$\Xi_{\rho_{1}}^{\rho_{2}}=\frac{\rho_{1} \rho_{2} \exp \left(\frac{m}{\Omega}\left(\rho_{1}^{2}+\rho_{2}^{2}\right)\right) m^{\frac{1}{2}-m}\left(\Gamma\left(m, \frac{m \rho_{2}^{2}}{\Omega}\right)-\Gamma\left(m, \frac{m \rho_{1}^{2}}{\Omega}\right)\right)}{f_{m} \sqrt{2 \pi \Omega}\left(\rho_{2} \rho_{1}^{2 m} \exp \left(\frac{m}{\Omega} \rho_{2}^{2}\right)+\rho_{1} \rho_{2}^{2 m} \exp \left(\frac{m}{\Omega} \rho_{1}^{2}\right)\right)}$,

where $m$ is the Nakagami- $m$ fading severity parameter and $\Gamma(.,$.$) is the incomplete Gamma function.$

\section{Multi-Interval ACD ( $K$-ACD) FunCtion}

For the $K$ non-overlapping intervals spanning the envelope range $0-\rho_{\max }$ of the received fading envelope, the multiinterval ACD or $K$-ACD function can be expressed as

$$
\Theta_{0}^{\rho_{\max }}(K)=\left[\Xi_{\rho_{0}=0}^{\rho_{1}}, \Xi_{\rho_{1}}^{\rho_{2}}, \cdots, \Xi_{\rho_{K-1}}^{\rho_{K}=\rho_{\max }}\right] .
$$

By setting the non-uniform widths $z_{k}$ of the $K$ ARoI intervals appropriately, the probability of attaining an identical ACD for the $K$ intervals can be maximized. For this task, after setting $\rho_{0}=0$ and $\rho_{K}=\rho_{\max }$, the remaining $K-1$ separating thresholds $\left(\rho_{1}, \cdots, \rho_{K}-1\right)$ can be determined by manipulating the set of expressions

$$
\Xi_{\rho_{0}=0}^{\rho_{1}}=\Xi_{\rho_{1}}^{\rho_{2}}=\cdots=\Xi_{\rho_{K-1}}^{\rho_{K}=\rho_{\max }}=\Psi
$$

where $\Psi$ is the constant ACD value across the $K$ intervals. For SKG quantization purposes, a suitable $K$ can be set to achieve $\Psi / T_{s} \geq 1$, i.e., one or more envelope sample falls 
TABLE I: PDF, CDF, LCR, and AFD for Rayleigh, Rice, and Nakagami- $m$ distributions [2]

\begin{tabular}{|l|c|c|c|c|}
\hline Distribution & PDF, $f(r)$ & CDF, $F_{r}(\rho)$ & LCR, $N_{r}(\rho)$ & AFD, $\tau_{r}(\rho)$ \\
\hline \hline Rayleigh & $\frac{2 r}{\Omega} \exp \left(\frac{-r^{2}}{\Omega}\right)$ & $1-\exp \left(-\frac{\rho^{2}}{\Omega}\right)$ & $\frac{\sqrt{2 \pi} f_{m} \rho}{\sqrt{\Omega}} \exp \left(-\frac{\rho^{2}}{\Omega}\right)$ & $\frac{\left(\exp \left(\rho^{2} / \Omega\right)-1\right) \sqrt{\Omega}}{\sqrt{2 \pi} f_{m} \rho}$ \\
\hline Rician & $\frac{2(k+1) r}{\Omega} \exp \left(-k-\frac{(k+1) r^{2}}{\Omega}\right)$ & $1-Q_{1}(\sqrt{2 k}$, & $\sqrt{2 \pi(k+1)} f_{m} \frac{\rho}{\Omega} \exp \left(-k-\frac{(k+1) \rho^{2}}{\Omega}\right)$ & $\frac{1-Q_{1}\left(\sqrt{2 k}, \sqrt{\frac{2(k+1) \rho^{2}}{\Omega}}\right)}{\sqrt{2 \pi(k+1)} f_{m} \rho I_{\circ}\left(2 \rho \sqrt{\frac{k(k+1)}{\Omega}}\right)}$ \\
& $\times I_{\circ}\left(2 r \sqrt{\frac{k(k+1)}{\Omega}}\right)$ & $\left.\sqrt{\frac{2(k+1) \rho^{2}}{\Omega}}\right)$ & $\times I_{\circ}\left(2 \rho \sqrt{\frac{k(k+1)}{\Omega}}\right)$ & $\times \Omega \exp \left(k+\frac{(k+1) \rho^{2}}{\Omega}\right)$ \\
\hline Nakagami- $m$ & $\frac{2 m^{m} r^{2 m-1}}{\Omega^{m} \Gamma(m)} \exp \left(-\frac{m r^{2}}{\Omega}\right)$ & $\frac{\Gamma\left(m, \frac{m \rho^{2}}{\Omega}\right)}{\Gamma(m)}$ & $\frac{\sqrt{2 \pi} f_{m}}{\Omega \Gamma(m)} m^{m-1 / 2} \rho^{2 m-1} \exp \left(-\frac{m \rho^{2}}{\Omega}\right)$ & $\frac{\Gamma\left(m, \frac{m \rho^{2}}{\Omega}\right) \Omega \exp \left(\frac{m \rho^{2}}{\Omega}\right)}{\sqrt{2 \pi} f_{m} m^{m-1 / 2} \rho^{2 m-1}}$ \\
\hline
\end{tabular}

in each quantization interval, on average. For given channel conditions $\Psi$ decreases with increasing $K$.

2-ACD Function Example: For $K=2$, the separating threshold $\rho_{1}$ that divides the received envelope range into two intervals of identical ACD, i.e., $\Xi_{0}^{\rho_{1}}=\Xi_{\rho_{1}}^{\rho_{\max }}$ can be determined as

$$
\frac{F_{r}\left(\rho_{1}\right)}{N_{r}\left(\rho_{1}\right)}=\frac{1-F_{r}\left(\rho_{1}\right)}{N_{r}\left(\rho_{1}\right)} .
$$

For a Rayleigh-distributed envelope, the separating threshold $\rho_{1}$ for the 2-ACD function can be expressed in closed-form as

$$
\rho_{1}=\sqrt{\Omega \ln 2}
$$

For a Rice-distributed envelope, the separating threshold $\rho_{1}$ for the 2-ACD function can be obtained by manipulating the expression

$$
1-2 Q_{1}\left(\sqrt{2 k}, \sqrt{\frac{2 \rho_{1}^{2}(k+1)}{\Omega}}\right)=0,
$$

where the value of $\rho_{1}$ can be obtained by numerical inversion of the Marcum Q-function [3]. Alternatively, a closed-form approximation to $\rho_{1}$ can be obtained by using the first order Marcum Q-function approximation [4].

For the Nakagami- $m$ distributed envelope, the separating threshold $\rho_{1}$ for the 2-ACD function can be obtained by manipulating the expression

$$
\Gamma(m)-2 \Gamma\left(m, \frac{m \rho_{1}^{2}}{\Omega}\right)=0 .
$$

Similarly, the separating thresholds for $K>2$ case can also be obtained.

\section{ApPliCATION ExAMPLES AND NUMERICAL EVALUATION}

This section discusses some applications of the proposed ACD metric and $K$-ACD function. Most notably, numerical evaluation of the proposed ACD metric-based non-uniform quantization (NUQ) is conducted in the context of SKG for PLS.

a) SKG for PLS: PLS is an emerging paradigm of augmenting secure communications at the physical layer of wireless communication systems. PLS techniques can be keybased or key-less. The generation of symmetric encryption keys from independent observations of the common wireless channel between the legitimate nodes circumvents the need to distribute secret keys between the legitimate nodes, which in turn enhances the system secrecy performance. The behaviour of the common reciprocal channel between the legitimate nodes (say Alice and Bob) is a function of their spatial positions and it cannot be measured by the eavesdropper/Eve for sufficiently rich scattering. Fig. 2 illustrates the system model and channel profiles observed independently at Alice and Bob within a channel coherence time $T_{c}$. The $K=4$ level quantization and corresponding 4-ACD function are also shown in the figure. Practically, differences between hardware and noise conditions of Alice and Bob lead to differences between their measurements of the ideally reciprocal channel. For example, the dissimilar channel envelope measurements can be modeled by a Gauss Markov model as [5] $h_{a b}=$ $\hat{\rho} h_{b a}+\sqrt{1-\hat{\rho}^{2}} \varepsilon$, where $\hat{\rho} \in[0,1]$ is the correlation coefficient between envelope measured at Bob $h_{a b}$ and envelope measured at Alice $h_{b a}$. Furthermore, $\varepsilon \sim \mathcal{N}(0,1)$ represents the Gaussian-distributed error in envelope measurement at Bob. The symmetric SKG algorithms aim to generate identical secret keys independently at Alice and Bob, despite their dissimilar channel measurements. The KGR, KAP, and KRP are some of the conventional metrics [5] employed to measure the effectiveness of SKG algorithms and they will also be used in the numerical results provided here. Among the several SKG algorithms proposed in the literature, a notable algorithm proposed by Ye et al. [6] uses for SKG only those contiguous envelope samples, whose sequence length exceeds some predefined threshold $\left(\Xi_{\mathrm{th}}\right)$. This algorithm increases the KAP at the cost of reduced KGR. However, a favorable tradeoff between KGR and KAP can be achieved by appropriate selection of $\Xi_{\mathrm{th}}$. For this task we propose to set the multilevel common ACD value as the acceptance/rejection threshold for sample excursions, i.e., $\Xi_{\text {th }}=\Psi$. This ACD-based quantization also leads to improved KRP as shown later by the presented numerical results.

The application of proposed ACD metric in 4-level NUQ for SKG algorithm of [6] is depicted in Fig. 3. The legitimate channels $h_{a b}$ and $h_{b a}$ are modeled as Rayleigh fading and $10^{5}$ channel samples are generated for obtaining the presented results. We assume that the legitimate nodes have prior knowledge of $\hat{\rho}$, which is set to $\hat{\rho}=0.84$ for the displayed results. As evident in the figure, the ACDbased quantization for SKG ensures performance improvement in terms of KGR and KRP, while maintaining the same KAP as that of the conventional uniform quantization (UQ) scheme. KRP performance offered by NUQ and ACD-based 


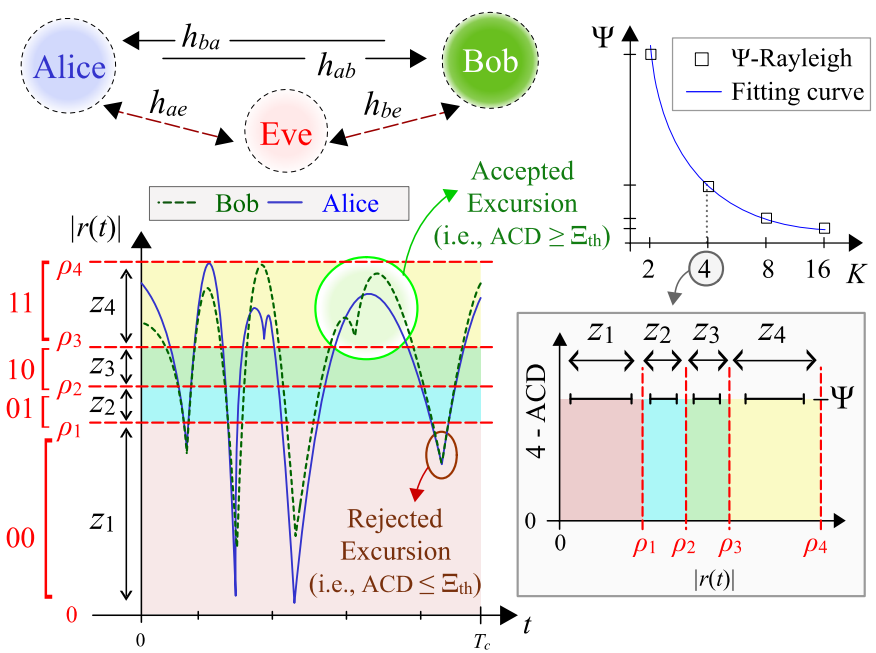

Fig. 2: $K$-ACD for SKG at Alice and Bob - PLS Example

NUQ is comparable. The NUQ scheme without ACD can provide promising KRP compared to UQ by maintaining same KGR and KAP [5] but the proposed ACD-based NUQ simultaneously improves both the KGR and KRP for the same KAP. This is due to the increased likelihood of identical length of contiguously located samples in each quantization interval in the ACD-based NUQ scheme. Fig. 3(b) shows the KRP evaluated by using the NIST test suite. The $P$ value is plotted for several NIST tests including the frequency, block frequency, run, discrete Fourier transform, Maurer, binary matrix rank, cumulative sum forward, and cumulative sum reversed tests for the proposed ACD-based NUQ and the conventional UQ schemes. As shown by Fig. 3(a), the performance improvement in KGR is more evident when the acceptance/rejection threshold $\Xi_{\text {th }}$ for sample excursions is set in accordance with the ACD metric, where $\hat{\Psi}=\operatorname{round}\left(\Psi / T_{s}\right)$. This indicates the scope for improving the SKG algorithms, e.g., [6] by utilizing the ACD information to further increase the KGR. A potential extension in the SKG algorithm can be the characterization of CD instances that qualify the minimum acceptance criteria (i.e., $\Xi_{\text {th }}$ ) into multiple CD length bins with codes of different sizes associated to each bin. This will result in the generation of more key-bits for longer $\mathrm{CD}$ instances and eventually result in further increased KGR.

b) Other Applications: The proposed ACD metric can be exploited to ensure high compression rate. For example, the number of $K$-ACD function-based NUQ intervals and sampling rate can be jointly optimized such that a minimal number of consecutive samples (ideally a single sample) fall in each quantization interval (i.e., each adjacent sample falls in different quantization intervals). Moreover, instead of encoding all the samples contiguously falling in a quantization interval, only the quantization interval index and ACD information can be used to encode the samples. A high compression rate can be achieved by interpreting the ACD behaviour of the signal under consideration to jointly devise appropriate values of the sampling rate, number and bounding thresholds of NUQ intervals. Furthermore, the code size associated with each quantization interval can also be manipulated by using the ACD information, e.g., ACD-based revisited Huffman coding.

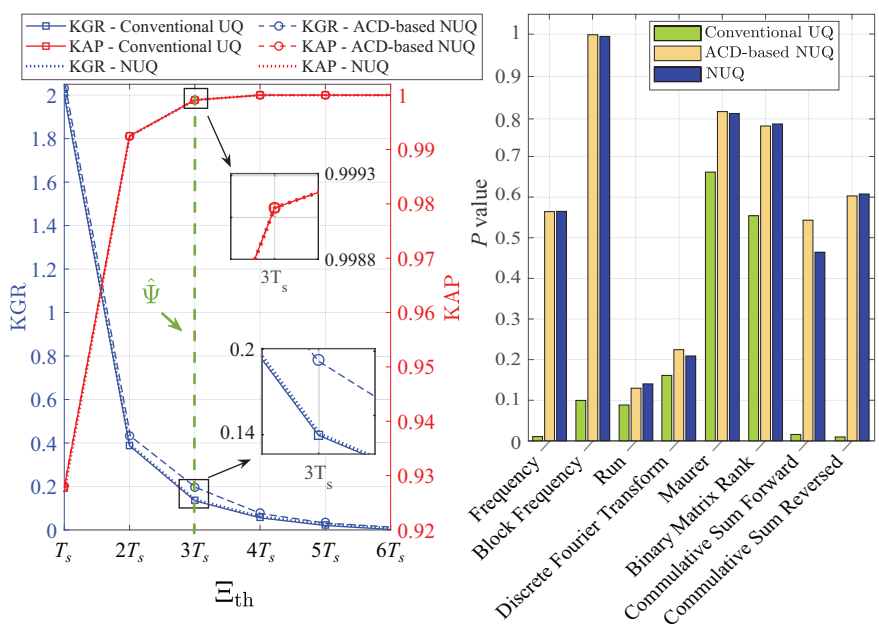

(a) KGR and KAP Performance

(b) KRP Performance (NIST test suite), $\Xi_{\mathrm{th}}=3 T_{S}$

Fig. 3: KGR, KAP, and KRP performance of 4-ACD quantization based SKG

Also, the proposed $K$-ACD function can be used for quantifying signal randomness. For example, a random number generator algorithm can be set to ensure a low ACD value for different amplitude intervals, for a given probability density function.

Angular spread quantification is critical in characterizing wireless channels and in the design of multi-antenna transceivers. Several angular spread quantifiers such as the true standard deviation are proposed in the literature [7]. The proposed ACD metric can be applied with modifications to the angular-domain for angular spread quantification.

\section{CONCLUSIONS}

In this letter, a novel ACD metric for the characterization of wireless fading channels has been proposed. Mathematical expressions of ACD metric for Rayleigh, Rice, and Nakagami$m$ distributions have been derived. A multi-level ACD (i.e., $K$ ACD) function has also been proposed. Various applications of the proposed ACD metric have been highlighted, where quantization for SKG has been numerically investigated.

\section{REFERENCES}

[1] A. F. Molisch, Wireless Communications. John Wiley \& Sons, 2011.

[2] A. Abdi, K. Wills, H. A. Barger, M. . Alouini, and M. Kaveh, "Comparison of the level crossing rate and average fade duration of Rayleigh, Rice and Nakagami fading models with mobile channel data," in proc. of IEEE Veh. Technol. Conf., vol. 4, 2000, pp. 1850-1857 vol.4.

[3] A. Gil, J. Segura, and N. M. Temme, "The asymptotic and numerical inversion of the marcum Q-function," Studies in Applied Mathematics, vol. 133, no. 2, pp. 257-278, 2014.

[4] M. Z. Bocus, C. P. Dettmann, and J. P. Coon, "An approximation of the first order Marcum Q-function with application to network connectivity analysis," IEEE Commun. Lett., vol. 17, no. 3, pp. 499-502, 2013.

[5] M. Adil, S. Wyne, and S. J. Nawaz, "On quantization for secret key generation from wireless channel samples," IEEE Access, vol. 9, pp. $21653-21668,2021$.

[6] C. Ye, S. Mathur, A. Reznik, Y. Shah, W. Trappe, and N. B. Mandayam, "Information-theoretically secret key generation for fading wireless channels," IEEE Trans. Inf. Forensics Security, vol. 5, no. 2, pp. 240254, 2010.

[7] S. J. Nawaz, N. M. Khan, and R. Ramer, "3-D spatial spread quantifiers for multipath fading wireless channels," IEEE Wireless Commun. Lett., vol. 5, no. 5, pp. 484-487, 2016. 